\title{
Method of Efficiently Predicting the Conducted Emissions of Multiple VSCS
}

\author{
P. M. Ivry ${ }^{1}$, O. A. $\mathrm{Oke}^{2}$, D.W.P. Thomas ${ }^{3}$, and M.Sumner ${ }^{4}$ \\ Electrical Systems and Optics Research Division \\ University of Nottingham \\ Nottingham, UK \\ 1 preyeivry@mail.ndu.edu.ng; ${ }^{2}$ oluwabukola_oke@ieee.org; ${ }^{3}$ dave.thomas@nottingham.ac.uk; ${ }^{4}$ mark.sumner@nottingham.ac.uk
}

\begin{abstract}
Various types of power converters including Voltage Source Converters (VSCs) have been known to produce conducted emissions. The net effect of simultaneously operating multiple VSCs is non-linear and this makes the prediction of the emission level more difficult. This paper presents an efficient method that can be used to predict conducted emissions of multiple VSCs in the presence of various uncertainties. It utilizes the Univariate Dimension Reduction (UDR) and the Bivariate Dimension Reduction (BDR) technique to predict the conducted emission level of multiple VSCs.
\end{abstract}

Keywords-Conducted Emissions; Dimension Reduction; Electromagnetic Compatibility; Prediction Method; Smart Grid; Uncertainty; Voltage Source Converter (VSC)

\section{INTRODUCTION}

Renewable energy generation is a key component of the smart grid and micro grid concept. Renewable energy sources usually utilize power electronic converters as an interface to any section of a power network. Power electronic converters generate conducted emissions at its switching frequency and multiples of its switching frequency. The conducted emissions are as a result of fast voltage switching action $(d v / d t)$ of the semiconductor devices [1]. These emissions have been known to increase transformer losses and cause equipment damage/malfunction. Particularly its interference with the proper operation of control and communication devices utilized in smart grids and microgrids. An example of the interference of the converters has been on smart meters. It was reported in [2] that conducted emissions from PV inverters has caused accuracy problems with smart meters where they operate in close proximity. It was suggested in [3] that an adequate assessment of harmonic emissions is required in smart grids to guarantee the compatibility between all equipment connected to the grid. Thus the Electromagnetic Compatibility (EMC) of the converter conducted emission is of grave concern for utilities, consumers and design engineers.

The level of conducted emissions generated by power converters are further increased by various uncertainties that may arise in its operation or at its design stage. Some of the factors have been listed in [4]. More so, the cumulative effect of simultaneously operating equipments that generate emissions (such as VSCs) is non-linear [5] and this makes the prediction of the emission level more difficult.
A common approach in predicting conducted emissions of power converters is by the use of Monte Carlo Simulation (MCS) [6], [7]. This approach requires tens of thousands of simulation to give an accurate prediction thus, making the MCS approach unfeasible for systems with large numbers of VSCs generating emissions.

This paper presents an efficient method that can be used to predict conducted emissions of multiple VSCs in the presence of various uncertainties. An enhanced Unscented Transform (UT) technique based on Univariate and Bivariate Dimension Reduction is utilized to achieve this. The paper is divided into five sections. The proposed Univariate and Bivariate Dimension Reduction technique is explained in section II. The modeled VSCs and the uncertainty are presented in Section III. Section IV presents and discusses the results and a conclusion is given in Section $\mathrm{V}$.

\section{UNSCENTED TRANSFORM AND DIMENSION REDUCTION TECHNIQUE}

\section{A. Unscented Transform (UT)}

The UT is an approximate estimator which works by approximating a nonlinear mapping by a set of selected points called sigma points. The weighted sum of the sigma points produces the expected value and variance of the mapping. The UT uses the moments of the PDF to develop the sigma points [8]. The UT has been utilized in nonlinear problems in electromagnetic compatibility[8], [9]. In [10] the unscented transform was described as an approximate estimator which works by approximating a continuous distribution function with PDF $\mathrm{w}(x)$ as a discrete distribution $w\left(x_{i}\right)$ using deterministically chosen points called sigma points $\left(S_{i}\right)$ such that the moments of both distributions are equal i.e.;

$$
E\left(x^{k}\right)=\int x^{k} w(x) d x=\sum_{i} w\left(x_{i}\right) S_{i}{ }^{k}
$$

Although the sigma points and weights may be obtained using Taylor's series expansion [8], its applicability is limited to few orders of approximation.

An alternative approach is to solve (1) as a quadrature problem such that the integration points correspond to the desired sigma points. For a function $f(x)$ with PDF (weighting function) $w(x)$, the nonlinear mapping for the

The first Author would like to acknowledge the Petroleum Technology Trust Fund (PTDF), Nigeria for sponsoring this research. 
expectation is given as;

$$
\sum_{i} w\left(x_{i}\right) f\left(S_{i}\right)=\int_{-\infty}^{\infty} f(x) w(x) d x=E[f(x)]
$$

Equation (2) is integrated using Gaussian quadrature in order to deduce the sigma points. The sigma points are obtained as the roots of the associated orthogonal polynomial.

\section{B. Sigma Point and Weight Estimation in Multivariate Problems}

The procedures discussed above is only applicable when obtaining individual sigma points and weights, for problems involving more than one random variable the simplest technique is based on tensor product of the individual sigma points. Unfortunately, this technique is plagued by a problem commonly referred to as the curse of dimensionality as the number of variables increase. For $n$ sigma points and $N$ number of variables the number of evaluations $E_{v}$ is given by:

$$
E_{V}=n^{N}
$$

For instance, 10 random variables with each discretized using 3 sigma points will need over 50,000 estimation points. Thus, the computational burden can exceed that of the MCS method. The method employed in [11] which is based on dimension reduction is therefore applied in this work for problems with more than one variable.

The dimension reduction [11-13] is a technique for function approximation so as to estimate the statistical moments of the output function. The technique involves an additive decomposition of an $N$-dimensional function involving $n$-dimensional integral into a series sum of $D$ dimensional functions such that $D<N$. For $\mathrm{D}=1$ the method is referred to as the univariate dimension reduction (UDR) while it is called bivariate dimension reduction (BDR) for $\mathrm{D}=2$. $\mathrm{A}$ detailed mathematical derivation of the techniques can be found in [12].

\section{Univariate Dimension Reduction (UDR)}

With the UDR, the main function, $f(\mathrm{x})$, is decomposed into a summation of one-dimensional functions such that;

$f(\mathbf{x})=\sum_{i=1}^{N} f\left(\mu_{1}, \cdots, \mu_{i-1}, x_{i}, \mu_{i+1}, \cdots, \mu_{N}\right)-(N-1) f\left(\mu_{1}, \cdots, \mu_{N}\right)$

where $\mu_{i}$ is the mean of the $i t h$ random variable.

The resultant function in (4) can easily be integrated since only one randomly distributed variable is present at every instance while the others are held constant at their mean values. The moments of the function are approximately the same as those of the decomposed function as represented in (5).

$$
E[f(\mathbf{x})] \approx E[\hat{f}(\mathbf{x})]=\sum_{i=1}^{N} E\left[f\left(\mu_{1}, \mu_{2}, \ldots, x_{i}, \mu_{N}\right)\right]-(N-1) E[f(\boldsymbol{\mu})]
$$

The same procedure is applied for getting the higher order moments.
The number of evaluations $\left(E_{V}\right)$ required for an $N$ dimensional function using $n$ estimation (sigma points) for the UDR is given in (6) [12].

$$
E_{V}=(n \times N)+1
$$

\section{Bivariate Dimension Reduction (BDR)}

The BDR produces a better representation of the real function since the approximation is truncated after the $2^{\text {nd }}$ order, although at the expense of computational time. Following a similar procedure to the UDR, the mean using the BDR can be evaluated using (7).

$$
\begin{gathered}
E[f(\mathbf{x})] \approx E[\hat{f}(\mathbf{x})]=\sum_{i<i 2}^{N} E\left[f\left(\mu_{1}, \ldots, x_{i 1}, \mu_{i+1}, \ldots, x_{i 2}, \ldots, \mu_{N}\right)\right]- \\
(N-2) \sum_{i=1}^{N} E\left[f\left(\mu_{1}, \mu_{2}, \ldots, x_{i}, \mu_{N}\right)\right]+\frac{(N-1)(N-2)}{2} E[f(\boldsymbol{\mu})]
\end{gathered}
$$

The BDR can thus be employed when accuracy is of higher priority than computational time. The number of evaluations $\left(E_{V}\right)$ required for an $\mathrm{N}$-dimensional function using $n$ estimation (sigma points) for the BDR is given in (8) [12].

$$
E_{v}=\frac{N(N-1)}{2} \times n^{2}+((n \times N)+1)
$$

The UDR and BDR $E_{v}$ is clearly a substantial saving in computational time over the alternative of $n^{N}$ evaluations.

\section{VSC STRUCTURE AND UNCERTAINTY}

The VSCs system studied and the uncertainty that might arise in the operation or design of the VSCs is discussed below.

\section{A. VSC Structure}

The studied VSC comprises DC Power Source, DC capacitor, VSC reactors, LCL filters, grid impedance and 3phase grid voltage (representing further parts of the grid). Fig. 1 shows two 2-level VSCs connected in parallel and interfaced to the grid at the PCC via the grid impedance. The system was simulated using PLECS ${ }^{\mathrm{TM}}$ [14] and MATLAB Simulink ${ }^{\mathrm{TM}}$ [15] simulation tools.

The switching pattern of the VSC is based on the Sinusoidal Pulse Width Modulation as in [16] and it is controlled based on the commonly used instantaneous power theory [17] using PI controllers.

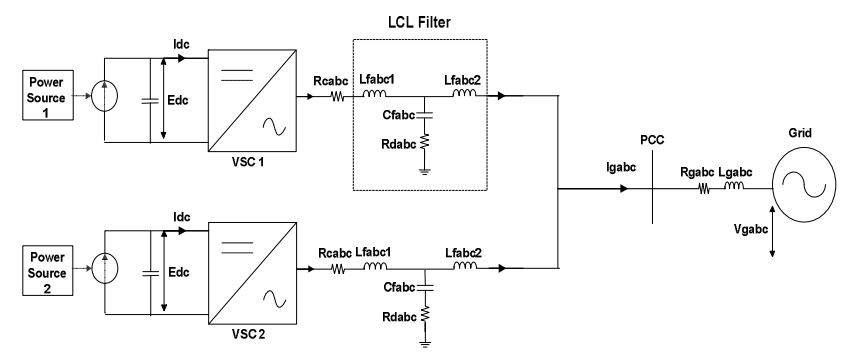

Figure 1. Shows the schematics of the studied VSC system 


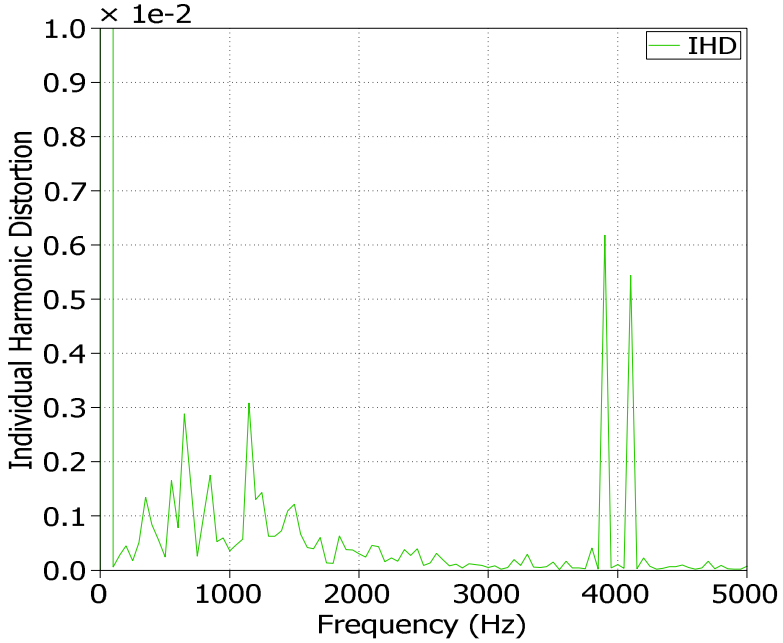

Figure 2. Shows the conducted emission at sidebands of the switching frequency relative to the fundamental frequency.

It was observed from Fig. 2 that high emissions are present at the sidebands of the VSC switching frequency $\left(f_{s w}=4 \mathrm{kHz}\right)$. These emissions are at the 78th and 82nd order.

\section{B. Uncertainty}

Three studies were considered in predicting the level of conducted emissions and they were based on the variation of the VSCs output power and the filter inductor value. In the first study, the effect of output power variations on the conducted emission is considered. The output power is assumed to vary randomly within a range following the uniform distribution between $25 \%$ and $100 \%$ of its rated power. The point here is to show wide variability of a renewable energy systems output power. The results are presented in Table I.

In the second study, the effect of filter inductor value $L_{f a b c l}$ on the level of conducted emission is investigated. For this system, $L_{f a b c l}$ was calculated using [18] and which gives a value of $5.76 \mathrm{mH}$. But there will nevertheless be some uncertainty as to the exact value chosen due to a trade-off between efficiency and cost. Based on this, and assuming the filter inductor value can be modeled using the uniform distribution, the variation range was chosen assuming a $\pm 40 \%$ trade-off between efficiency and cost. The statistical moments of the conducted emissions are presented in Table II.

The third study considers varying both the operating power of the VSC and the filter inductance $L_{f a b c l}$. The variations are within the values used in the first and second study. This is done to investigate the combined effect of filter design choice and output power variation on the level of conducted emissions of multiple VSCs. The results obtained for each of the cases are discussed in the sections below.

\section{PREDICTED RESUlts}

\section{A. Power Variation}

It can be seen from Table I that the BDR result has a closer agreement with the MCS for all moments presented than the UDR. The prediction of the skewness and kurtosis of the
VSCs combined current THD using the BDR has a $42 \%$ and $45 \%$ error respectively. The UDR results are close to the MCS for its mean and standard deviation however, the skewness and kurtosis are totally inaccurate.

TABLE I. UNCERTAINTY DUE TO POWER VARIATION

\begin{tabular}{|c|c|c|c|c|}
\hline Moments & THD/IHD & MCS & UDR & BDR \\
\hline \multirow{3}{*}{ Mean } & THD & 3.429 & 3.814 & 3.582 \\
\cline { 2 - 5 } & 78th & 0.431 & 0.430 & 0.432 \\
\cline { 2 - 5 } & 82nd & 0.364 & 0.362 & 0.365 \\
\hline \multirow{3}{*}{ Std } & THD & 1.030 & 1.145 & 1.146 \\
\cline { 2 - 5 } & 78th & 0.129 & 0.102 & 0.134 \\
\cline { 2 - 5 } & 82nd & 0.111 & 0.087 & 0.114 \\
\hline \multirow{3}{*}{ Skewness } & THD & 1.031 & -0.621 & 0.589 \\
\cline { 2 - 5 } & 78th & 1.529 & -0.070 & 1.518 \\
\cline { 2 - 5 } & 82nd & 1.522 & -0.095 & 1.559 \\
\hline \hline \multirow{3}{*}{ Kurtosis } & THD & 4.789 & 1.432 & 2.659 \\
\cline { 2 - 5 } & 78th & 5.754 & 1.120 & 5.486 \\
\cline { 2 - 5 } & 82nd & 5.655 & 1.146 & 5.651 \\
\hline
\end{tabular}

\section{B. Filter Variation}

From Table II it is observed that the results of the BDR technique in predicting the conducted emission are accurate for the mean and standard deviation. Here again the BDR method under predicted the VSCs current THD skewness and kurtosis by a $30 \%$ and $22 \%$ error respectively. The UDR method efficiently predicts the mean and standard deviation of the VSCs THD and conducted emission at the 78th and 82nd order. In this case the UDR accuracy is better for the skewness and kurtosis however; the emissions are either under or over predicted.

TABLE II. UNCERTAINTY DUE TO FILTER VARIATION

\begin{tabular}{|c|c|c|c|c|}
\hline Moments & THD/IHD & MCS & UDR & BDR \\
\hline \multirow{3}{*}{ Mean } & THD & 1.892 & 1.963 & 1.873 \\
\cline { 2 - 5 } & 78th & 0.286 & 0.285 & 0.290 \\
\cline { 2 - 5 } & 82nd & 0.241 & 0.241 & 0.245 \\
\hline \hline \multirow{3}{*}{ Std } & THD & 0.284 & 0.232 & 0.250 \\
\cline { 2 - 5 } & 78th & 0.067 & 0.068 & 0.072 \\
\cline { 2 - 5 } & 82nd & 0.055 & 0.057 & 0.060 \\
\hline \hline \multirow{3}{*}{ Skewness } & THD & 0.812 & -0.987 & 0.566 \\
\cline { 2 - 5 } & 78th & 0.400 & 0.430 & 0.462 \\
\cline { 2 - 5 } & 82nd & 0.387 & 0.429 & 0.192 \\
\hline \hline \multirow{3}{*}{ Kurtosis } & THD & 3.416 & 2.409 & 2.654 \\
\cline { 2 - 5 } & 78th & 2.432 & 1.940 & 2.481 \\
\cline { 2 - 5 } & 82nd & 2.430 & 1.888 & 2.405 \\
\hline
\end{tabular}

\section{Power and Filter Variation}

The combined effect of the power and filter variation on the conducted emission was again well predicted by the BDR method. Although it is not as accurate as the results obtained in the other two cases The UDR was only successful in 
predicting the mean and standard deviation of the conducted emission and THD.

TABLE III. UNCERTAINTY DUE TO POWER AND FILTER VARIATION

\begin{tabular}{|c|c|c|c|c|}
\hline Moments & THD/IHD & MCS & UDR & BDR \\
\hline \multirow{3}{*}{ Mean } & THD & 3.537 & 3.299 & 3.494 \\
\cline { 2 - 5 } & 78th & 0.476 & 0.518 & 0.519 \\
\cline { 2 - 5 } & 82nd & 0.401 & 0.441 & 0.439 \\
\hline \multirow{3}{*}{ Std } & THD & 1.229 & 1.585 & 1.749 \\
\cline { 2 - 5 } & 78th & 0.197 & 0.230 & 0.292 \\
\cline { 2 - 5 } & 82nd & 0.166 & 0.194 & 0.248 \\
\hline \hline \multirow{3}{*}{ Skewness } & THD & 1.175 & 0.201 & 1.391 \\
\cline { 2 - 5 } & 78th & 1.892 & 0.088 & 1.621 \\
\cline { 2 - 5 } & 82nd & 1.916 & 0.055 & 1.627 \\
\hline \hline \multirow{3}{*}{ Kurtosis } & THD & 5.562 & 1.015 & 5.401 \\
\cline { 2 - 5 } & 78th & 8.939 & 1.493 & 6.093 \\
\cline { 2 - 5 } & 82nd & 9.166 & 1.507 & 6.140 \\
\hline
\end{tabular}

If only the mean and standard deviation are required then the UDR method can be seen to be very reliable. As it requires fewer numbers of evaluations and provides significant savings in computational time.

\section{CONCLUSION}

An efficient method for predicting conducted emissions of multiple VSCs using univariate and bivariate dimension reduction method have been investigated. The UDR and BDR methods require less number of evaluations than the MCS. Majority of the results showed that the BDR method has a good agreement with the MCS approach. The mean and standard deviation results are very close to the MCS for the UDR and the BDR. However, the UDR failed to reliably give an accurate prediction of higher moments like the skewness and kurtosis. For statistical analysis of conducted emissions where only the mean and standard deviation is required the UDR would be a very good option. The BDR accurately predicts the higher moment however, this is done at the expense of computational burden and time with the increase of the number of evaluations.

\section{REFERENCES}

[1] H. Bishnoi, A. C. Baisden, P. Mattavelli, and D. Boroyevich, "Analysis of EMI Terminal Modeling of Switched Power Converters," IEEE Trans. Power Electronics, vol. 27, pp. 3924-3933, 2012.

[2] P. F. Keebler, "Meshing power quality and electromagnetic compatibility for tomorrow's smart grid," IEEE Electromagnetic Compatibility Magazine, vol. 1, pp. 100-103, 2012.

[3] Bollen M. H. J., Zhong J., Zavoda F., Meyer J., McEachern A., and L. F. C., "Power Quality aspects of Smart Grids," Inter. Conf. on Renewable Energies and Power Quality (ICREPQ), Granada, 2010.
[4] P. M. Ivry, M. J. Rawa, D. W. P. Thomas, and M. Sumner, "Power quality of a voltage source converter in a smart grid," in Proc. IEEE PowerTech Conf., Grenoble, 2013, pp. 1-6.

G. F. Bartak and A. Abart, "EMI of emissions in the frequency range $2 \mathrm{kHz}-150 \mathrm{kHz}$," in Proc. Inter. Conf. and Exhibition on Electricity Distribution (CIRED), 2013, pp. 1-4.

H. Junping, J. Jianguo, H. Jiangjiang, and C. Wei, "Model of EMI coupling paths for an off-line power converter," in Proc. IEEE Applied Power Electronics Conf. and Expo., 2004, vol.2, pp. 708-713

M. Ferber, C. Vollaire, L. Krahenbuhl, J. L. Coulomb, and J. A. Vasconcelos, "Conducted EMI of DC-DC Converters With Parametric Uncertainties," IEEE Trans. Electromagnetic Compatibility, vol. 55, pp. 699-706, 2013.

L. R. A. X. De Menezes, D. W. P. Thomas, C. Christopoulos, A. Ajayi, and P. Sewell, "The use of Unscented Transforms for statistical analysis in EMC," in Proc. IEEE Inter. Symp. Electromagnetic Compatibility - EMC Europe, 2008, pp. 1-5.

L. R. A. X. De Menezes, A. Ajayi, C. Christopoulos, P. Sewell, and G. A. Borges, "Efficient computation of stochastic electromagnetic problems using unscented transforms," IET Science, Measurement \& Technology, vol. 2, pp. 88-95, 2008.

[10] O. A. Oke, D. W. P. Thomas, and G. M. Asher, "A new probabilistic load flow method for systems with wind penetration," in Proc. IEEE PowerTech Conf., Trondheim, 2011, pp. 1-6.

[11] O. A. Oke, "Enhanced Unscented Transform Method for Probabilistic Load Flow Studies," Ph.D. dissertation, Dept. Electrical and Electronics Eng., University of Nottingham, Nottingham, 2013.

[12] H. Xu and S. Rahman, "A generalized dimensionreduction method for multidimensional integration in stochastic mechanics," Inter. Journal for Numerical Methods in Eng., vol. 61, pp. 1992-2019, 2004.

[13] B. Huang and X. Du, "Uncertainty Analysis by Dimension Reduction Integration and Saddlepoint Approximations," Journal of Mechanical Design, vol. 128, pp. 26-33, 2005.

[14] PLECS 3.4.1, Plexim GmbH, Zurich, 2013.

[15] MATLAB 7.12.0 (R2011a), MathWorks, Inc., 2011.

[16] N. Mohan, T. M. Undeland, and W. P. Robbins, Power Electronics, Converters, Applications and Design, 3rd ed.: John Wiley and Sons, 2003.

[17] A. Yazdani and R. Iravani, Voltage-Source Converters in Power Systems: Modelling, Control and Applications: John Wiley and Sons, 2010.

[18] K. H. Ahmed, S. J. Finney, and B. W. Williams, "Passive Filter Design for Three-Phase Inverter Interfacing in Distributed Generation," in Proc. Compatibility in Power Electronics. 2007, pp. 1-9. 